\title{
Probing the Diffuse Optical-IR Background with TeV Blazars Detected with the MAGIC Telescopes
}

\author{
Elisa Prandini ${ }^{1 *}$, Alberto Domínguez ${ }^{2}$, Vandad Fallah Ramazani ${ }^{3}$, Tarek Hassan ${ }^{4}$, \\ Daniel Mazin ${ }^{5,6}$, Abelardo Moralejo ${ }^{4}$, Mireia Nievas Rosillo' ${ }^{2}$, Gaia Vanzo ${ }^{7,8}$, and \\ Monica Vazquez Acosta ${ }^{7,8}$ for the MAGIC Collaboration \\ ${ }^{1}$ Dipartimento di Fisica e Astronomia "G. Galilei", University of Padova, Padua, Italy, ${ }^{2}$ Departamento de Física Atómica, \\ Universidad Complutense, Madrid, Spain, ${ }^{3}$ Tuorla observatory, University of Turku, Turku, Finland, ${ }^{4}$ IFAE, The Barcelona \\ Institute of Science and Technology, Bellaterra, Spain, ${ }^{5}$ Max Planck Institute for Physics, Munich, Germany, ${ }^{6}$ Institute for \\ Cosmic Ray Research, University of Tokyo, Tokyo, Japan, ${ }^{7}$ Instituto de Astrofísica de Canarias, Tenerife, Spain, \\ ${ }^{8}$ Departamento de Astrofisica, Universidad de La Laguna, Tenerife, Spain
}

Blazars are radio loud quasars whose jet points toward the observer. The observed emission is mostly non-thermal, dominated by the jet emission, and in some cases extends up to the very high energy gamma rays (VHE; E > $100 \mathrm{GeV}$ ). To date, more than 60 blazars have been detected at VHE mainly with ground-based imaging atmospheric Cherenkov telescopes (IACTs) such as MAGIC, H.E.S.S., and VERITAS. Energetic photons from a blazar may interact with the diffuse optical and IR background (the extragalactic background light, EBL) leaving an imprint on the blazar energy spectrum. This effect can be used to constrain the EBL, with basic assumptions on the intrinsic energy spectrum. Current generation of IACTs is providing valuable measurements of the EBL density and energy spectrum from optical to infrared frequencies. In this contribution, we present the latest results obtained with the data taken with the MAGIC telescopes: using 32 spectra from 12 blazars, the scale factor of the optical density predicted by the EBL model from Domínguez et al. (2011) is constrained to be $0.95(+0.11,-0.12)_{\text {stat }}$ $(+0.16,-0.07)_{\text {sys }}$, where a value of 1 means the perfect match with the model.

Keywords: Active Galactic Nuclei (AGN), blazars, Extragalactic Background Light (EBL), relativistic jets, MAGIC telescopes, gamma rays

\section{THE EXTRAGALACTIC BACKGROUND LIGHT AND ITS IMPRINT IN BLAZAR SPECTRA}

Received: 22 October 2017 Accepted: 07 November 2017 Published: 22 November 2017

Citation:

Prandini E, Domínguez A, Fallah Ramazani V, Hassan T, Mazin D, Moralejo A, Nievas Rosillo M, Vanzo G and Vazquez Acosta M for the MAGIC

Collaboration (2017) Probing the Diffuse Optical-IR Background with TeV Blazars Detected with the MAGIC Telescopes.

Front. Astron. Space Sci. 4:50. doi: 10.3389/fspas.2017.00050
The optical-infrared diffuse background that permeates the Universe is also referred as the Extragalactic Background Light (EBL). The EBL consists of light emitted by stars directly (optical) and, in part, absorbed by dust in their host galaxies and re-emitted at longer wavelengths (IR) since the birth of the first stars.

A collection of current measurements of the EBL is described in Cooray (2016), a recent and detailed review on EBL measurements and applications. Direct measurements of the EBL are complex due to strong foreground radiations from our Galaxy, but mainly from our own Solar System. Solid lower limits on the EBL density are provided by galaxy counts. Different models which take into account the evolution of galaxies have been proposed to explain and predict this 2012). They differ from the degree of complexity and approach, but all converge on a similar optical and IR background (e.g., Franceschini et al., 2008; Domínguez et al., 2011; Gilmore et al., 
spectral shape and intensity for the EBL. Yet, some differences persist in the cosmic evolution of the background.

A limited number of extragalactic sources (approximately 60) is known to emit electromagnetic radiation up to the so-called very high energies (VHE, E > $100 \mathrm{GeV}$ ). The large majority of these sources are blazars, active galaxies displaying a jet of ultra-relativistic particles almost aligned to the line of sight. Main engine of the activity is an accreting, supermassive black hole. Blazars emitting at VHE offer a unique opportunity to test EBL models. Gamma rays with energies of hundreds of $\mathrm{GeV}$ and above may be, in fact, absorbed due to the interaction with EBL photons via electron-positron pair creation (Salamon and Stecker, 1998). The absorption is not only a distance dependent effect but also an energy dependent effect. Due to the energy dependence of the pair production, we have an

TABLE 1 | List of 12 blazars observed with the MAGIC telescopes and used in this study, sorted from the lowest to the highest redshifts.

\begin{tabular}{llcc}
\hline Source name & Source type & Redshift & Observation time [h] \\
\hline Markarian 421 & HBL & 0.03 & 43.8 \\
(15 spectra) & & & \\
1ES 1959+650 & HBL & 0.048 & 4.8 \\
OT 546 & HBL & 0.055 & 6.4 \\
BL Lacertae & IBL & 0.069 & 1.0 \\
1ES 0229+200 & HBL & 0.14 & 105.2 \\
1ES 1011+496 & HBL & 0.212 & 11.8 \\
PKS 1510-089 & FSRQ & 0.361 & 5.0 \\
(2 spectra) & & & \\
PKS 1222+216 & FSRQ & 0.432 & 0.5 \\
PG 1553+113 & HBL & $0.43-0.58$ & 66.4 \\
(5 spectra) & & & \\
PKS 1424+240 & HBL & 0.604 & 49.1 \\
(2 spectra) & & & \\
PKS 1441+25 & FSRQ & 0.939 & 2.1 \\
QSO B0218+35 & FSRQ & 0.944 &
\end{tabular}

imprint of the EBL in the observed spectrum from a blazar. This imprint on the blazar spectra may be used to constrain the EBL itself, under some assumptions on the shape of the intrinsic spectra.

The study presented in this paper aims at testing the state-ofthe-art EBL models by means of a sample of 32 blazar spectra spanning a redshift from 0.03 to 0.944 collected with the MAGIC telescopes.

\section{THE MAGIC TELESCOPES}

MAGIC (Major Atmospheric Gamma Imaging Cherenkov) is a system of two Imaging Atmospheric Cherenkov Telescopes (IACTs) designed to observe VHE gamma rays from $50 \mathrm{GeV}$ up to tens of TeV (Aleksić et al., 2016a). It is located in the Canary island of La Palma, at $\sim 2,200 \mathrm{~m}$ above the sea level. Since 2004, MAGIC has been used to observe and detect a significant signal from tens of blazars located in the Northern hemisphere. For a recent review, see Sitarek (2017).

MAGIC achieved his best performances after 2012, when the last major upgrade of the system took place. The current integral sensitivity above $220 \mathrm{GeV}$ is $(0.66 \pm 0.03) \%$ of the Crab Nebula flux in $50 \mathrm{~h}$ of observations, when assuming point-like sources with Crab Nebula-like spectrum. The angular resolution at those energies, defined as the sigma of a 2-dimensional Gaussian distribution, is $<0.07$ degree. The energy resolution is $16 \%$ and is defined as the standard deviation obtained from a Gaussian fit to the distribution of (Eest - Etrue)/Etrue, where Eest is the reconstructed energy and Etrue is the true energy simulated with Monte Carlo data. More details on the data analysis and the MAGIC performance can be found in Aleksić et al. (2016b).

\section{METHODS}

The purpose of this study is to use the largest available dataset collected with the MAGIC telescopes and set new limits on the EBL energy density. In particular, we adopt the method proposed
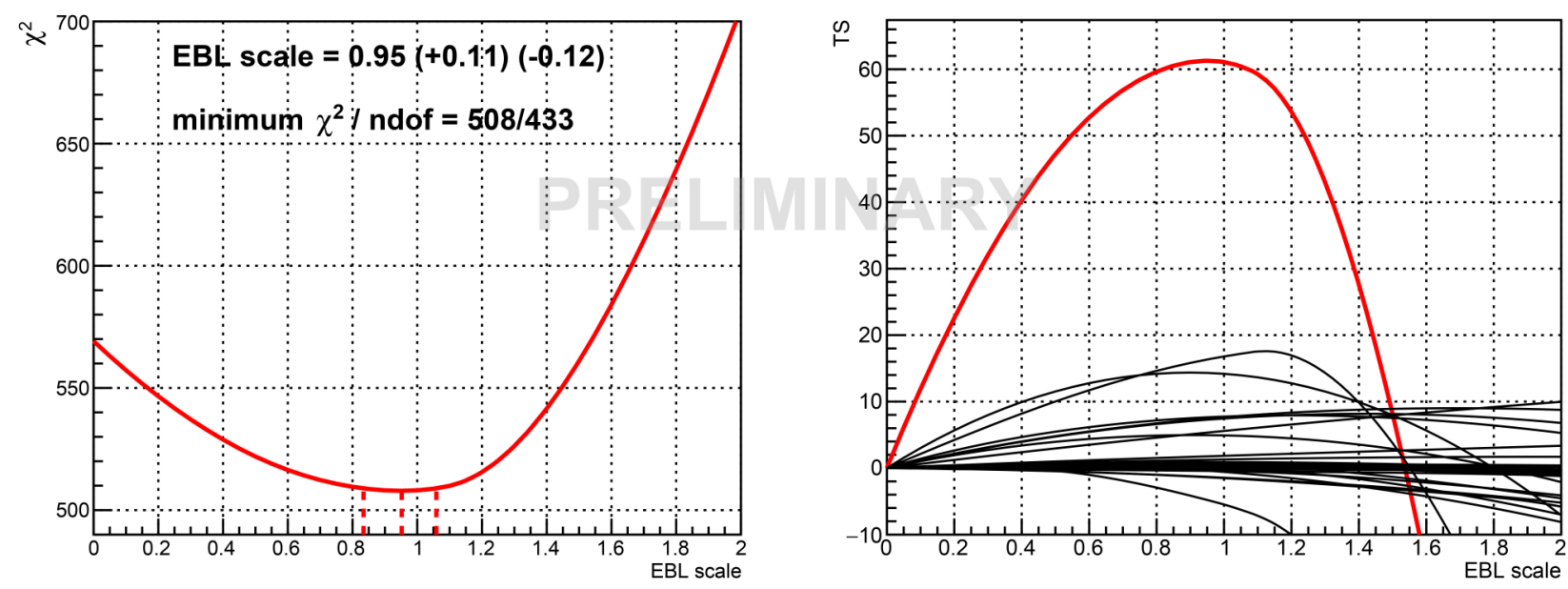

FIGURE 1 | Left: Total $\chi^{2}$ from the maximum likelihood fit. Right: Total (red) and spectrum-wise (black) Test Statistics TS $=\chi^{2}(0)-\chi^{2}$ vs. EBL scale relative to the Domínguez model. 


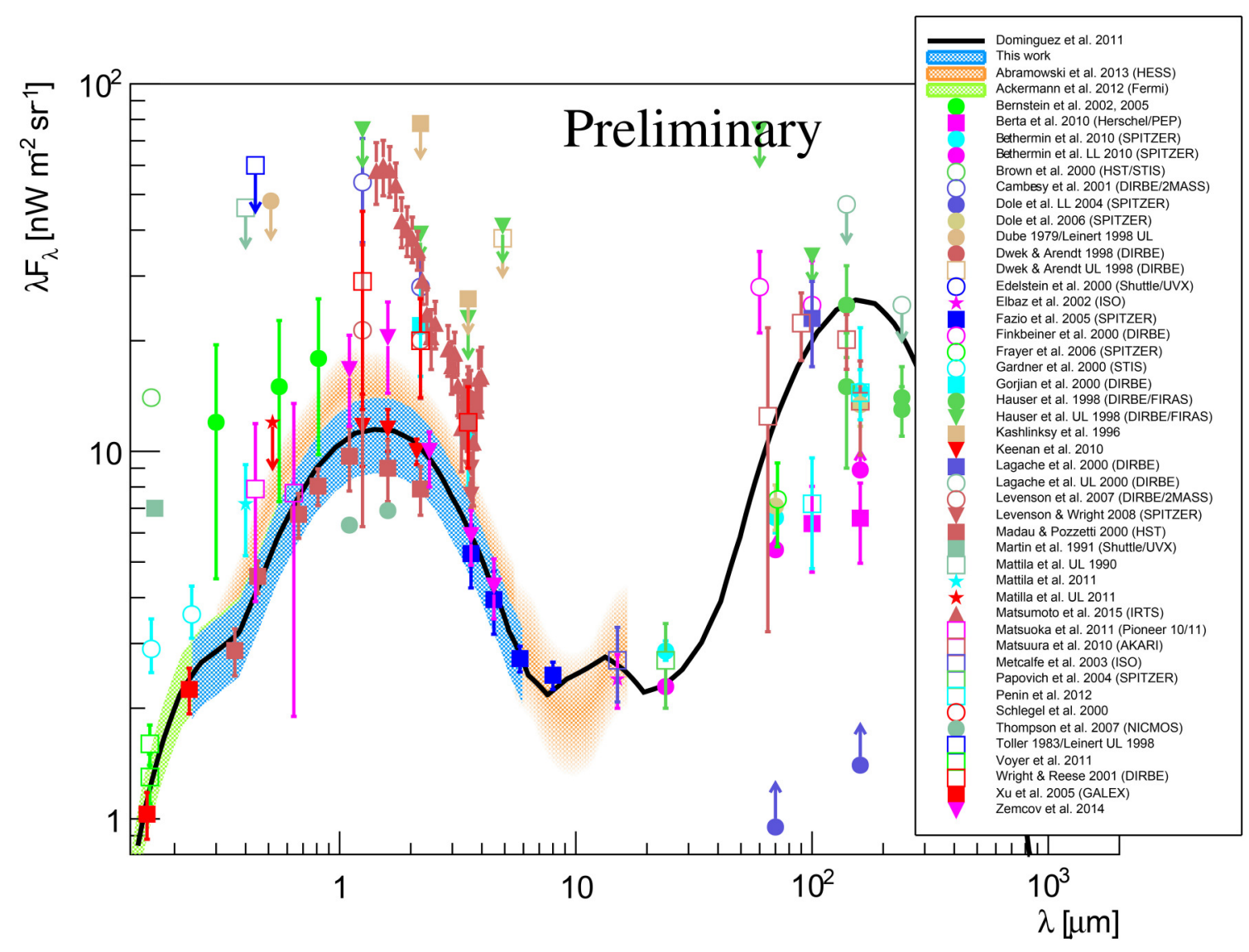

FIGURE 2 | A collection of EBL measurements with our measurements (blue area). The reported uncertainties are statistical only.

in Abramowski et al. (2013) which consists in estimating a scale factor for the EBL energy density, and can be outlined as follows:

1. Assume a particular EBL spectral shape and its evolution over redshift. For this work we have considered the model from Domínguez et al. (2011);

2. Assume an instrinsic shape that could describe the blazar spectrum before any interaction with the EBL. To this purpose, we have taken into account the following shapes: logparabola, power-law with exponential cut-off, log-parabola with exponential cut-off, and power-law with sub/super exponential cut-off;

3. In order to estimate the most likely EBL scale factor, we have then adopted the poissonian maximum likelihood approach. We have compared the measured number of events in bins of estimated energy with the expected number of events estimated by assuming a particular instrinsic spectrum folded with the MAGIC instrument response function and corrected for the EBL absorption effect $\left(\mathrm{dF} / \mathrm{dE}_{\text {observed }}=\mathrm{dF} / \mathrm{dE}_{\text {intrinsic }}\right.$. $\left.e^{-\alpha \tau(E, z)}\right)$. Free parameters are the EBL scale factor (alpha) and the intrinsic spectral parameters. The cosmic-ray background rates vs. estimated energy are treated as nuisance parameters.
4. The previous step has been iteratively repeated in order to scan the EBL scale factor and the different instrinsic spectral shapes. In each step the fit probability has been used to decide among the 4 spectral shape options and the best fit $\chi^{2}$ was estimated. The scale $\alpha_{0}$ for which the $\chi^{2}$ reaches its minimum $\left(\chi_{\min }^{2}\right)$ is the value which best fits the data, and the condition $\Delta \chi^{2}=1$ relative to the minimum indicates the $1 \sigma$ statistical uncertainty range.

\section{DATASET}

To perform this study, we have used 32 spectra from 12 sources, summarized in Table 1. The use, in some cases, of multiple spectra from the same source is due to the variability of the observed spectrum. The sources considered belong to two different blazar classes (second column, from TeVCat ${ }^{1}$ ): flat spectrum radio quasars (FSRQs) and high frequency peaked BL Lac objects (HBL). They span a redshift range from 0.03 to 0.944 , third column. The observation time of MAGIC is reported in the last column of the Table.

\footnotetext{
${ }^{1}$ http://tevcat2.uchicago.edu
} 


\section{RESULTS}

The results of our study are displayed in Figure 1. On the left panel the total $\chi^{2}$, which is the linear combination of the best $\chi^{2}$ values from the individual spectra, from the maximum likelihood fit procedure applied to each spectrum is reported. The minimum $\chi^{2}$ corresponds to a scale factor $\alpha_{0}=0.95_{-0.12}^{+0.11}$.

The right panel of Figure 1 displays the corresponding Test Statistics (TS $\left.=\chi^{2}(0)-\chi^{2}\right)$ of the total and for each individual spectrum curves as a function of the EBL scale relative to the Domínguez model.

Figure 2 shows a compilation of EBL data and models, plus the results of our analysis. This figure also shows the model proposed by Domínguez et al. (2011) whose spectral intensity is multiplied by our scale factor $\alpha_{0}=0.95_{-0.12}^{+0.11}$. It is in good agreement with recent EBL measurements (Abramowski et al., 2013; Sitarek, 2017).

\subsection{Systematic Uncertainties}

In order to evaluate the systematic uncertainties affecting our estimate, we have allowed for a maximum average deviation of $\pm 15 \%$ between data and Monte Carlo simulations of the gammaray showers and the detector in terms of deviation in the light throughput (atmosphere and telescopes), and tested its effect on the EBL estimate. Moreover, we have also considered the effect introduced by the different assumptions of the intrinsic spectral function. Our result is the best-fit EBL scale, $\alpha_{0}=$ $0.95\left({ }_{-0.12}^{+0.11}\right)_{\text {stat }}\left({ }_{-0.15}^{+0.19}\right)_{s y s}$.

\section{CONCLUSIONS}

Observations of blazars with the MAGIC telescopes have been used to estimate new constraints on the EBL energy density. The strategy adopted is based on the maximum-likelihood fit to 32 VHE spectra taken with the MAGIC telescopes, in analogy with similar studies carried out using the High Energy Stereoscopic System, H.E.S.S. (Abramowski et al., 2013), and Fermi-Large Area Telescope (LAT) data (Ackermann et al., 2012). The method consists in estimating the most likely EBL scale factor, once an evolving EBL spectral shape, and an intrinsic blazar spectrum are assumed.

\section{REFERENCES}

Abramowski, A., Acero, F., Aharonian, F., Akhperjanian, A. G., Anton, G., Balenderan, S., et al. (2013). Measurement of the extragalactic background light imprint on the spectra of the brightest blazars observed with H.E.S.S. Astron. Astrophys. 550:A4. doi: 10.1051/0004-6361/2012 20355

Ackermann, M., Ajello, M., Allafort, A., Schady, P., Baldini, L., Ballet, J., et al. (2012). The imprint of the extragalactic background light in the gamma-ray spectra of blazars. Science 338:1190. doi: 10.1126/science.12 27160

Aleksić, J., Ansoldi, S., Antonelli, L. A., Antoranz, P., Babic, A., Bangale, P., et al. (2016a). The major upgrade of the MAGIC telescopes, Part I: The hardware improvements and the commissioning of the
In this work, we have assumed the EBL model from Domínguez et al. (2011), and allowed different possible shapes for the intrinsic spectrum (log-parabola, power-law with exponential cut-off, log-parabola with exponential cut-off, and power-law with sub/super exponential cut-off). For the EBL scale factor we have obtained $\alpha_{0}=0.95\left({ }_{-0.12}^{+0.11}\right)_{\text {stat }}\left({ }_{-0.15}^{+0.19}\right)_{s y s}$, which is fully consistent with other constraints and with state-of-the-art EBL models.

\section{AUTHOR CONTRIBUTIONS}

EP contributed to the data analysis and wrote the manuscript. $\mathrm{AM}$ and DM initiated and coordinated the project, contributed to the data analysis, and provided a critical review of the manuscript. AD, VF, TH, MN, GV, and MV contributed to the data analysis and provided a critical review of the manuscript.

\section{FUNDING}

This work has been partially supported by University of Padova.

\section{ACKNOWLEDGMENTS}

We would like to thank the Instituto de Astrofísica de Canarias for the excellent working conditions at the Observatorio del Roque de los Muchachos in La Palma. The financial support of the German BMBF and MPG, the Italian INFN and INAF, the Swiss National Fund SNF, the ERDF under the Spanish MINECO (FPA2015-69818-P, FPA2012-36668, FPA201568378-P, FPA2015-69210-C6-2-R, FPA2015-69210-C6-4-R, FPA2015-69210-C6-6-R, AYA2015-71042-P, AYA2016-76012C3-1-P, ESP2015-71662-C2-2-P, CSD2009-00064), and the Japanese JSPS and MEXT is gratefully acknowledged. This work was also supported by the Spanish Centro de Excelencia "Severo Ochoa" SEV-2012-0234 and SEV-2015-0548, and Unidad de Excelencia "María de Maeztu" MDM-2014-0369, by the Croatian Science Foundation (HrZZ) Project 09/176 and the University of Rijeka Project 13.12.1.3.02, by the DFG Collaborative Research Centers SFB823/C4 and SFB876/C3, the Polish National Research Centre grant UMO-2016/22/M/ST9/00382 and by the Brazilian MCTIC, CNPq, and FAPERJ.

system. Astropart. Phys. 72, 61-75. doi: 10.1016/j.astropartphys.2015. 04.004

Aleksić, J., Ansoldi, S., Antonelli, L. A., Antoranz, P., Babic, A., Bangale, P., et al. (2016b). The major upgrade of the MAGIC telescopes, Part II: A performance study using observations of the Crab Nebula. Astropart. Phys. 72, 76-94. doi: 10.1016/j.astropartphys.2015.02.005

Cooray, A. (2016). Extragalactic background light measurements and applications. Roy. Soc. Open Sci. 3:150555. doi: 10.1098/rsos.150555

Domínguez, A., Primack, J. R., Rosario, D. J., Prada, F., Gilmore, R. C., Faber, S. M., et al. (2011). Extragalactic background light inferred from AEGIS galaxy-SED-type fractions. Month. Notices R. Astron. Soc. 410, 2556-2578. doi: 10.1111/j.1365-2966.2010.17631.x

Franceschini, A., Rodighiero, G., and Vaccari, M. (2008). Extragalactic optical-infrared background radiation, its time evolution and the 
cosmic photon-photon opacity. Astron. Astrophys. 487, 837-852. doi: 10.1051/0004-6361:200809691

Gilmore, R. C., Somerville, R. S., Primack, J. R., and Domínguez, A. (2012). Semi-analytic modelling of the extragalactic background light and consequences for extragalactic gamma-ray spectra. Month. Notices R. Astron. Soc. 422, 3189-3207. doi: 10.1111/j.1365-2966.2012. 20841.x

Salamon, M. H., and Stecker, F. W. (1998). Absorption of high-energy gamma rays by interactions with extragalactic starlight photons at high redshifts and the high-energy gamma-ray background. Astrophys. J. 493, 547-554. doi: $10.1086 / 305134$

Sitarek, J. (2017). Highlights of the MAGIC AGN program. ArXiv e-prints.
Conflict of Interest Statement: The authors declare that the research was conducted in the absence of any commercial or financial relationships that could be construed as a potential conflict of interest.

Copyright (c) 2017 Prandini, Dominguez, Fallah Ramazani, Hassan, Mazin, Moralejo, Nievas Rosillo, Vanzo, and Vazquez Acosta for the MAGIC Collaboration. This is an open-access article distributed under the terms of the Creative Commons Attribution License (CC BY). The use, distribution or reproduction in other forums is permitted, provided the original author(s) or licensor are credited and that the original publication in this journal is cited, in accordance with accepted academic practice. No use, distribution or reproduction is permitted which does not comply with these terms. 